\title{
Two variables generalized Laguerre polynomials
}

\author{
Asad A.
}

\begin{abstract}
The objective of this paper is to introduce and study the generalized Laguerre polynomial for two variables. We prove that these polynomials are characterized by the generalized hypergeometric function. An explicit representation, generating functions and some recurrence relations are shown. Moreover, these polynomials appear as solutions of some differential equations.

Key words and phrases: Laguerre polynomial, hypergeometric function, generating function, recurrence relation.
\end{abstract}

University of Agriculture, Faisalabad, Pakistan

E-mail: mrasadali5100@gmail.com

\section{Introduction}

Laguerre polynomials play a great role in mathematics and in mathematical physics. They can be found in many monographs on special functions. Special functions are particular mathematical functions which have more or less established names and notations due to their importance in mathematical analysis, functional analysis, physics or other branches of mathematics. There is no need for us to review the impact that classical orthogonal polynomial and special functions theory have applications in mathematics, science, engineering and computations $[1,4,12]$. Laguerre, Hermite, Bateman, Gegenbauer and Chebyshev polynomial sequences have appeared in connection with the study of differential equations $[2,3,6]$. In [5,7], the Laguerre and Hermite polynomials were introduced as examples of right orthogonal polynomial sequences for appropriate right moment functionals of integral type. The Laguerre polynomials were introduced and studied in [8]. In [9], it is shown that these polynomials are orthogonal with respect to a weight function. Recently, the numerical inversion of Laplace transforms using Laguerre polynomials has been given in [10]. A generalized form of the Bateman polynomials is presented in $[3,9]$. The Laguerre polynomials for two variables are defined in [7] by the generating function

$$
\sum_{n=0}^{\infty} L_{n}^{(\alpha)}(x, y) t^{n}=(1-y t)^{-1-\alpha} e^{\left(\frac{-x t}{1-y t}\right)} .
$$

By using [5], we can get the following equation

$$
L_{n}^{(\alpha)}(x, y)=\sum_{k=o}^{n} \frac{(1+\alpha)_{n}(-x)^{k} y^{n-k}}{(n-k) !(1+\alpha)_{k} k !} .
$$


From (2) we can easily derive

$$
L_{n}^{(\alpha)}(x, y)=\frac{(1+\alpha)_{n} y^{n}}{n !}{ }_{1} F_{1}\left(-n ; 1+\alpha ; \frac{x}{y}\right)
$$

where $n \in \mathbb{N}_{0}, 1+\alpha \in \mathbb{C} \backslash \mathbb{Z}_{0}^{-}, x, y \in \mathbb{C}$. Here and elsewhere, let $\mathbb{N}, \mathbb{R}$ and $\mathbb{C}$ be the sets of positive integers, real number and complex numbers, respectively, and let $\mathbb{N}_{0}:=\mathbb{N} \cup\{0\}$. ${ }_{1} F_{1}$ is a particular case of the well-known generalized hypergeometric series ${ }_{p} F_{q}, p, q \in \mathbb{N}_{0}$, given by [13, p. 73]

$$
{ }_{p} F_{q}\left[\begin{array}{l}
\alpha_{1}, \ldots, \alpha_{p} ; \\
\beta_{1}, \ldots, \beta_{q} ;
\end{array}\right]=\sum_{n=0}^{\infty} \frac{\left(\alpha_{1}\right)_{n} \cdots\left(\alpha_{p}\right)_{n}}{\left(\beta_{1}\right)_{n} \cdots\left(\beta_{q}\right)_{n}} \frac{z^{n}}{n !}={ }_{p} F_{q}\left(\alpha_{1}, \ldots, \alpha_{p} ; \beta_{1}, \ldots, \beta_{q} ; z\right) .
$$

Here $(\lambda)_{v}$ denotes the Pochhammer symbol, which is defined for $\lambda, v \in \mathbb{C}$ in terms of the familiar Gamma function $\Gamma$ by

$$
(\lambda)_{v}:=\frac{\Gamma(\lambda+v)}{\Gamma(\lambda)}= \begin{cases}1, & v=0, \lambda \in \mathbb{C} \backslash\{0\} \\ \lambda(\lambda+1) \cdots(\lambda+n-1), & v=n \in \mathbb{N}, \lambda \in \mathbb{C} .\end{cases}
$$

It being read traditionally that $(\lambda)_{0}:=1$. The particular case $\alpha=0$ of (3), i.e.

$$
L_{n}(x, y)=L_{n}^{(0)}(x, y)=y^{n} F_{1}\left(-n ; 1 ; \frac{x}{y}\right)
$$

where $n \in \mathbb{N}_{0}, x, y \in \mathbb{C}$, is called simple Laguerre polynomial for two variables, which has also accepted much attention. Numerous generating functions can produce (3) or (6), certain formulas and properties, including these polynomials $[1,9,11,13]$.

\section{Two variables generalized Laguerre polynomials}

We begin by defining two variables generalized Laguerre polynomials (TVGLP for short) $L_{p, n}^{(\alpha)}(x, y)$ by the following generating function

$$
\frac{1}{(1-y t)^{1+\alpha}} \exp \left(\frac{-x^{p} t^{p}}{(1-y t)^{p}}\right)=\sum_{n=0}^{\infty} L_{n, p}^{(\alpha)}(x, y) t^{n}, \quad p \in \mathbb{N} ; x, y, \alpha \in \mathbb{C} .
$$

One may observe that for $p=1$ the relations (1) and (7) are identical. That is, $L_{n, 1}^{(\alpha)}(x, y)=$ $L_{n}^{(\alpha)}(x, y)$, which is classical Laguerre polynomials for two variables. Hereafter we explore certain formulas and properties involving the TVGLP in (7). Throughout, let $F(p ; x, y, t)$ be the left-handed generating function in (7). 


\subsection{Explicit expression}

We give an explicit expression of TVGLP $L_{n, p}^{(\alpha)}(x, y)$ and prove that these polynomials are characterized by the generalized hypergeometric function in the following theorem.

Theorem 1. Let $x, \alpha \in \mathbb{C}, p \in \mathbb{N}$, and $n \in \mathbb{N}_{0}$. Then

$$
\begin{aligned}
L_{n, p}^{(\alpha)}(x, y) & =(1+\alpha)_{n} \sum_{k=0}^{[n / p]} \frac{(-1)^{k} y^{n-p k}}{k !(1+\alpha)_{p k}(n-p k) !} x^{p k} \\
& =\frac{(1+\alpha)_{n}}{n !} y^{n} \sum_{k=0}^{[n / p]} \frac{(-1)^{(p+1) k}(-n)_{p k}}{k !(1+\alpha)_{p k}}\left(\frac{x}{y}\right)^{p k} .
\end{aligned}
$$

Here and throughout, $[m]$ denotes the greatest integer less than or equal to $m \in \mathbb{R}$. Or, equivalently,

$$
L_{n, p}^{(\alpha)}(x, y)=\frac{(1+\alpha)_{n}}{n !} y_{p}^{n} F_{p}\left[\begin{array}{c}
\frac{-n}{p}, \frac{-n+1}{p}, \ldots, \frac{-n-1+p}{p} ; \\
\frac{\alpha+1}{p}, \frac{\alpha+2}{p}, \ldots, \frac{\alpha+p}{p} ;
\end{array}\right.
$$

Proof. Expanding the exponential in the left-hand side of (7), we find

$$
F(p ; x, y, t)=\frac{1}{(1-y t)^{1+\alpha+p k}} \sum_{k=0}^{\infty} \frac{(-1)^{k} x^{p k} t^{p k}}{k !} .
$$

Employing the binomial theorem

$$
(1-z)^{-a}=\sum_{n=0}^{\infty} \frac{(a)_{n}}{n !} z^{n}={ }_{1} F_{0}(a ;-; z), \quad a \in C,|z|<1,
$$

we obtain the following double series

$$
F(p ; x, y, t)=\sum_{n=0}^{\infty} \sum_{k=0}^{\infty} \frac{(-1)^{k}(1+\alpha+p k)_{n} y^{n} x^{p k}}{k ! n !} t^{n+p k}
$$

Recall a known double series manipulation [13]:

$$
\sum_{n=0}^{\infty} \sum_{k=0}^{\infty} A(k, n)=\sum_{n=0}^{\infty} \sum_{k=0}^{[n / p]} A(k, n-p k), \quad p \in \mathbb{N}
$$

is equivalent to

$$
\sum_{n=0}^{\infty} \sum_{k=0}^{[n / p]} A(k, n)=\sum_{n=0}^{\infty} \sum_{k=0}^{\infty} A(k, n+p k), \quad p \in \mathbb{N},
$$

where $A$ denotes a function of two variables and the involved double series is assumed to be absolutely convergent. 
Applying (12) in (11), we get

$$
F(p ; x, y, t)=\sum_{n=0}^{\infty} \sum_{k=0}^{[n / p]} \frac{(-1)^{k}(1+\alpha+p k)_{n-p k} y^{n-p k} x^{p k}}{k !(n-p k) !} t^{n}
$$

Equating the coefficients of $t^{n}$ in the right members of (7) and (14) yields

$$
L_{n, p}^{(\alpha)}(x, y)=\sum_{k=0}^{[n / p]} \frac{(-1)^{k}(1+\alpha+p k)_{n-p k}}{k !(n-p k) !} y^{n-p k} x^{p k}
$$

Using (5) and a known identity

$$
(n-k) !=\frac{(-1)^{k} n !}{(-n)_{k}}, \quad k, n \in N_{0}, 0 \leq k \leq n
$$

we derive

$$
(1+\alpha+p k)_{n-p k}=\frac{(1+\alpha)_{n}}{(1+\alpha)_{p k}} \quad \text { and } \quad(n-p k) !=\frac{(-1)^{p k} n !}{(-n)_{p k}} .
$$

Hence, using (16) in (15) leads to the desired identity (8). Finally, applying the multiplication formula

$$
(\lambda)_{m n}=m^{m n} \prod_{j=1}^{m}\left(\frac{\lambda+j-1}{m}\right)_{n}, \quad \lambda \in \mathbb{C}, m \in \mathbb{N}, n \in \mathbb{N}_{0},
$$

to (8), gives the equivalent expression (9).

\subsection{Generating functions}

We establish two generating functions for TVGLP $L_{n, p}^{(\alpha)}(x, y)$ in the following theorem.

Theorem 2. The two variables generalized Laguerre polynomials satisfy the following generating functions

$$
e_{0}^{y t} F_{p}\left(-; \frac{\alpha+1}{p}, \frac{\alpha+2}{p}, \ldots, \frac{\alpha+p}{p} ;-\left(\frac{x t}{p}\right)^{p}\right)=\sum_{n=0}^{\infty} \frac{L_{n, p}^{(\alpha)}(x, y) t^{n}}{(1+\alpha)_{n}}
$$

and

$$
\frac{1}{(1-y t)^{c}} p F_{p}\left(\begin{array}{c}
\frac{c}{p}, \frac{c+1}{p}, \ldots, \frac{c+p-1}{p} ; \\
\frac{\alpha+1}{p}, \frac{\alpha+2}{p}, \ldots, \frac{\alpha+p}{p} ;
\end{array}-\left(\frac{x t}{1-y t}\right)^{p}\right)=\sum_{n=0}^{\infty} \frac{(c)_{n} L_{n, p}^{(\alpha)}(x, y) t^{n}}{(1+\alpha)_{n}}, \quad|t|<1
$$


Proof. Using (8), (13), and (17), we have

$$
\sum_{n=0}^{\infty} \frac{L_{n, p}^{(\alpha)}(x, y) t^{n}}{(1+\alpha)_{n}}=\sum_{n=0}^{\infty} \frac{(y t)^{n}}{n !} \sum_{k=0}^{\infty} \frac{(-1)^{k}(x t)^{p k}}{k !(1+\alpha)_{p k}}=e^{y t} \sum_{k=0}^{\infty} \frac{(-1)^{k}}{k ! \prod_{j=1}^{p}\left(\frac{\alpha+j}{p}\right)_{k}}\left(\frac{x t}{p}\right)^{p k}
$$

In view of (4), the rightmost term of (20) can be expressed as the left-hand side of (18). Employing (8), (13), and (10), we find

$$
\begin{aligned}
\sum_{n=0}^{\infty} \frac{(c)_{n} L_{n, p}^{(\alpha)}(x, y) t^{n}}{(1+\alpha)_{n}} & =\sum_{k=0}^{\infty} \sum_{n=0}^{\infty} \frac{(c+p k)_{n}(y t)^{n}}{n !} \cdot \frac{(c)_{p k}\left\{-(x t)^{p}\right\}^{k}}{k !(1+\alpha)_{p k}} \\
& =\frac{1}{(1-y t)^{c}} \sum_{k=0}^{\infty} \frac{(c)_{p k}}{k !(1+\alpha)_{p k}}\left\{-\left(\frac{x t}{1-y t}\right)^{p}\right\}^{k}
\end{aligned}
$$

which, upon using (17) and (4), leads to the left-hand member of (19).

It is noted that the case $c=1+\alpha$ of (19) yields the generating function (7).

\subsection{Recurrence relations}

We give some recurrence relations involving TVGLP $L_{n, p}^{(\alpha)}(x, y)$ and their derivative in the following theorem.

Theorem 3. The two variables generalized Laguerre polynomials satisfy the following relations

$$
\begin{gathered}
x D L_{n, p}^{(\alpha)}(x, y)-n L_{n, p}^{(\alpha)}(x, y)+y(\alpha+n) L_{n-1, p}^{(\alpha)}(x, y)=0, \\
D L_{n, p}^{(\alpha)}(x, y)= \begin{cases}0, & 0 \leq n \leq p-1, \\
-p x^{p-1} L_{n-p, p}^{(\alpha+p)}(x, y), & n \geq p,\end{cases} \\
y(\alpha+n) L_{n-1, p}^{(\alpha)}(x, y)-n L_{n, p}^{(\alpha)}(x, y)=p x^{p} L_{n-p, p}^{(\alpha+p)}(x, y), \quad n \geq p,
\end{gathered}
$$

where $D=\frac{d}{d x}$.

Proof. From (20), we can set

$$
G(p ; x, y, t):=\sum_{n=0}^{\infty} \frac{L_{n, p}^{(\alpha)}(x, y) t^{n}}{(1+\alpha)_{n}}=e^{y t} \Phi\left(\frac{-x^{p} t^{p}}{p^{p}}\right)
$$

where

$$
\Phi\left(\frac{-x^{p} t^{p}}{p^{p}}\right)=\sum_{k=0}^{\infty} \frac{(-1)^{k}}{k ! \prod_{j=1}^{p}\left(\frac{\alpha+j}{p}\right)_{k}}\left(\frac{x t}{p}\right)^{p k}
$$


Differentiating $G(p ; x, y, t)$ with respect to $x$ and $t$, respectively, gives

$$
G_{x}(p ; x, y, t)=e^{y t} \Phi^{\prime}\left(\frac{-x^{p} t^{p}}{p^{p}}\right) \cdot \frac{-x^{p-1} t^{p}}{p^{p-1}}
$$

and

$$
G_{t}(p ; x, y, t)=y e^{y t} \Phi\left(\frac{-x^{p} t^{p}}{p^{p}}\right)+e^{y t} \Phi^{\prime}\left(\frac{-x^{p} t^{p}}{p^{p}}\right) \cdot \frac{-x^{p} t^{p-1}}{p^{p-1}} .
$$

Combining $G_{x}(p ; x, y, t)$ and $G_{t}(p ; x, y, t)$ yields

$$
x G_{x}(p ; x, y, t)-t G_{t}(p ; x, y, t)+y t G(p ; x, y, t)=0 .
$$

Applying the series in (24) to (25), we obtain

$$
\sum_{n=1}^{\infty} \frac{x D L_{n, p}^{(\alpha)}(x, y) t^{n}}{(1+\alpha)_{n}}-\sum_{n=1}^{\infty} \frac{n L_{n, p}^{(\alpha)}(x, y) t^{n}}{(1+\alpha)_{n}}+y \sum_{n=1}^{\infty} \frac{L_{n-1, p}^{(\alpha)}(x, y) t^{n}}{(1+\alpha)_{n-1}}=0
$$

We find from (26) that each coefficient of $t^{n}$ should be zero, which gives (21).

Differentiating both sides of (7) provides

$$
\begin{aligned}
\sum_{n=1}^{\infty} D L_{n, p}^{(\alpha)}(x, y) t^{n} & =\frac{1}{(1-y t)^{1+\alpha+p}} \exp \left(\frac{-x^{p} t^{p}}{(1-y t)^{p}}\right) \cdot\left(-p x^{p-1} t^{p}\right) \\
& =-p x^{p-1} \sum_{n=0}^{\infty} L_{n, p}^{(\alpha+p)}(x, y) t^{n+p} \\
& =-p x^{p-1} \sum_{n=p}^{\infty} L_{n-p, p}^{(\alpha+p)}(x, y) t^{n},
\end{aligned}
$$

which, upon equating the coefficients of $t^{n}, n \geq p$, in the leftmost and rightmost members, produces (22). Setting (22) in (21) provides (23).

Theorem 4. The two variables generalized Laguerre polynomials is solution of the following differential equation

$$
\left[\frac{1}{p} \theta \prod_{j=1}^{p}\left(\frac{1}{p}(\theta-1+\alpha+j)\right)-(-1)^{p+1}\left(\frac{x}{y}\right)^{p} \prod_{j=1}^{p} \frac{1}{p}(\theta+j-n-1)\right] L_{n, p}^{(\alpha)}(x, y)=0,
$$

where $\theta=x \frac{d}{d x}$.

Proof. With the suggestion of equation (9) before us, we can proceed as follows

$$
\begin{aligned}
& \phi=\frac{(1+\alpha)_{n}(y)^{n}}{n !}{ }_{p} F_{p}\left(\frac{-n}{p}, \frac{-n+1}{p} \ldots\right.\left.\frac{-n+p-1}{p} ; \frac{1+\alpha}{p}, \frac{2+\alpha}{p} \ldots \frac{p+\alpha}{p} ;(-1)^{p+1}\left(\frac{x}{y}\right)^{p}\right) \\
&=\frac{(1+\alpha)_{n}}{n !} \sum_{k=0}^{\left[\frac{n}{p}\right]} \frac{\prod_{i=1}^{p}\left(\frac{i-n-1}{p}\right)_{k}(-1)^{(p+1) k} x^{p k} y^{n-p k}}{\prod_{i=1}^{p}\left(\frac{i+\alpha}{p}\right)_{k} k !} .
\end{aligned}
$$


Since $\frac{1}{p} \theta x^{p k}=k x^{p k}$, it follows that

$$
\left[\frac{1}{p} \theta \prod_{j=1}^{p}\left(\frac{1}{p}(\theta-1+\alpha+j)\right)\right] \phi=\frac{(1+\alpha)_{n}}{n !} \sum_{k=1}^{\left[\frac{n}{p}\right]} \frac{\prod_{i=1}^{p}\left(\frac{i-n-1}{p}\right)_{k}\left(\frac{i+\alpha+k-1}{p}\right)(-1)^{(p+1) k} x^{p k} y^{n-p k}}{\prod_{i=1}^{p}\left(\frac{i+\alpha}{p}\right)_{k}(k-1) !} .
$$

But the last factor in $\left(\frac{i+\alpha}{p}\right)_{k}$ is $\left(\frac{i+\alpha+k-1}{p}\right)$, so the above equality can be proceed as follows

$$
\frac{(1+\alpha)_{n}}{n !} \sum_{k=1}^{\left[\frac{n}{p}\right]} \frac{\prod_{i=1}^{p}\left(\frac{i-n-1}{p}\right)_{k}(-1)^{(p+1) k} x^{p k} y^{n-p k}}{\prod_{i=1}^{p}\left(\frac{i+\alpha}{p}\right)_{k-1}(k-1) !} .
$$

Now we replace $k$ by $k+1$ and have

$$
\begin{gathered}
{\left[\frac{1}{p} \theta \prod_{i=0}^{p} \frac{1}{p}(\theta-1+\alpha+i)\right] \phi=\frac{(1+\alpha)_{n}}{n !} \sum_{k=0}^{\left[\frac{n}{p}\right]} \frac{\prod_{i=1}^{p}\left(\frac{i-n-1}{p}\right)_{k+1}(-1)^{(p+1)(k+1)} x^{p(k+1)} y^{n-p(k+1)}}{\prod_{i=1}^{p}\left(\frac{i+\alpha}{p}\right)_{k} k !}} \\
=(-1)^{p+1}\left(\frac{x}{y}\right)^{p} \frac{(1+\alpha)_{n}}{n !} \sum_{k=0}^{\left[\frac{n}{p}\right]} \frac{\prod_{i=1}^{p}\left(\frac{i-n-1}{p}\right)_{k} \prod_{i=1}^{p}\left(\frac{k+i-n-1}{p}\right)(-1)^{(p+1) k} x^{p k} y^{n-p k}}{\prod_{i=1}^{p}\left(\frac{i+\alpha}{p}\right)_{k} k !} \\
=(-1)^{p+1}\left(\frac{x}{y}\right)^{p}\left[\prod_{j=1}^{p} \frac{1}{p}(\theta+j-n-1)\right] \phi .
\end{gathered}
$$

Thus, we have shown that $\phi$ is solution of differential equation. It is worth noticing that taking $p=1$ in (27) gives the following

$$
\left(x \frac{d^{2}}{d x^{2}}+\left((1+\alpha)-\frac{x}{y}\right) \frac{d}{d x}+\frac{n}{y}\right) L_{1, n}^{(\alpha)}(x, y)=0,
$$

which is the differential of classical Laguerre polynomials for two variables.

\section{Acknowledgements}

The author express their sincere gratitude to the editor and unknown referee for carefully reading the manuscript and for their valuable comments and suggestions which greatly improved this paper. 


\section{References}

[1] Andrews G.E., Askey R., Roy R. Special Functions, Cambridge University Press, 2004.

[2] Asad A. A generalization of the Laguerre polynomials. Commun. Korean Math. Soc. 2021, 36 (2), $299-312$. doi:10.4134/CKMS.c200208

[3] Asad A., Muhammad Z.I., Bilal A., Ather M. Generalization of Bateman Polynomials. Int. J. Anal. App. 2019,17 (5), 803-808. doi:10.28924/2291-8639-17-2019-803

[4] Asad A., Mujahid I., Aqeela N., Zaib U.N. Solution of Fractional k-Hypergeometric Differential Equation. Int. J. Math. Anal. 2020, 14 (3), 125-132. doi:10.12988/ijma.2020.91287

[5] Bedratyuk L., Bedratuyk A. The inverse and derivative connecting problems for some Hypergeometric polynomials. Carpathian Math. Publ. 2018, 10 (2), 235-247. doi:10.15330/cmp.10.2.235-247

[6] Romdhane N.B. A General Theorem on Inversion Problems for Polynomial Sets. Mediterr. J. Math. 2016, 13, 2783-2793. doi:10.1007/s00009-015-0654-8

[7] Dattoli G., Torre A. Operational methods and two-variable, Laguerre polynomials. Atti Accad. Sci. Torino CI. Sci. Fis. Math. Natur. 1998, 132, 1-7.

[8] Hendriksen E. A weight function for the associated Jacobi-Laurent polynomials. J. Comput. Appl. Math. 1990, 33 (2), 171-180. doi:10.1016/0377-0427(90)90367-9

[9] Karlsson P.W. Hypergeometric functions with integral parameter differences. J. Math. Phys. 1971, 12 (2), $270-271$. doi:10.1063/1.1665587

[10] Koepf W., Schmersau D. Representations of orthogonal polynomials. J. Comput. Appl. Math. 1998, 90 (1), 57-94. doi:10.1016/S0377-0427(98)00023-5

[11] Minton B.M. Generalized hypergeometric function of unit argument. J. Math. Phys. 1970, 11 (4), 1375-1376. doi: 10.1063/1.1665270

[12] Slater L.J. Generalized Hypergeometric functions. Cambridge University Press, Cambridge, 1966.

[13] Rainville E.D. Special Functions. The Macmillan Company, New York, 1960.

Received 05.04.2020

Revised 18.08.2020

Асад А. Узагальнені поліноми Аагерра від двох змінних // Карпатські матем. публ. - 2021. - Т.13, №1. - С. 134-141.

Метою цієї статті $є$ представлення та вивчення узагальнених поліномів $\Lambda$ агерра від двох змінних. Ми доводимо, що ці поліноми характеризуються узагальненою гіпергеометричною функцією. Показано явне представлення, генеруючу функцію та деякі рекурентні співвідношення. Більше того, ці поліноми з'являються як розв'язки деяких диференціальних рівнянь.

Ключові слова $і$ фрази: поліном Аагерра, гіпергеометрична функція, генеруюча функція, рекурентне співвідношення. 